\title{
Fish consumption and self-reported physical and mental health status
}

\author{
Karen M Silvers ${ }^{1, *}$ and Kate M Scott ${ }^{2}$ \\ ${ }^{1}$ New Zealand Institute for Crop \& Food Research, Private Bag 11600 , Palmerston North, New Zealand: \\ ${ }^{2}$ Department of Psychological Medicine, Wellington School of Medicine, New Zealand
}

Submitted 28 November 2000: Accepted 21 June 2001

\begin{abstract}
Objective: The aim of this study was to assess whether self-reported mental health status, measured using the SF-36 questionnaire, was associated with fish consumption, assessed using a food-frequency questionnaire.

Design: The cross-national data were collected in the 1996/97 New Zealand Health Survey and 1997 Nutrition Survey, which were conducted using the same sampling frame. Survey respondents were categorised into those who consumed no fish of any kind and those who consumed some kind of fish, at any frequency. Data were adjusted for age, household income, eating patterns, alcohol use and smoking. Other demographic variables and potential confounding nutrients were included in the preliminary analyses but were not found to have a significant relationship with fish consumption.

Subjects: Data from a nationally representative sample of 4644 New Zealand adults aged 15 years and over were used in this analysis.

Results: Fish consumption was significantly associated with higher self-reported mental health status, even after adjustment for possible confounders. Differences between the mean scores for fish eaters and those who never eat fish were 8.2 for the Mental Health scale $(P=0.005)$ and 7.5 for the Mental Component score $(P=0.001)$. Conversely, the association between fish consumption and physical functioning was in the opposite direction $(P=0.045)$.

Conclusions: This is the first cross-sectional survey to demonstrate a significant relationship between fish intake and higher self-reported mental health status, therefore offering indirect support for the hypothesis that $\omega-3$ polyunsaturated fatty acids may act as mood stabilisers.

Keywords

Fish

Diet

Polyunsaturated fatty acids Self-reported mental health status Depression
\end{abstract}

There is now evidence from population ${ }^{1}$, case- control $^{2,3}$ and experimental studies ${ }^{4}$ which indicates that $\omega-3$ polyunsaturated fatty acids (PUFAs) may be of aetiological importance in mood disorders. During this century there has been a significant increase in the lifetime risk of major depression ${ }^{5,6}$. Klerman and Weissman ${ }^{6}$ found that the increase was not explained by changing diagnostic criteria, changing attitudes of health professionals and societies, reporting bias, differential mortality, institutional or other artefacts. However, the increase in risk does correlate with a change in diet, which includes a higher intake of $\omega-6$ PUFAs and a lower intake of $\omega-3$ PUFAs $^{7-9}$. Similarly, these changes in diet are thought to have contributed to the significant increase in cardiovascular disease and inflammatory disorders this century ${ }^{9,10}$. Interestingly, depression is the strongest psychological predictor of coronary heart disease ${ }^{11}$.

Dietary sources of $\omega-3$ PUFAs include many plants, phytoplankton and algae, and all animals that have consumed them, either on land or in the sea. The basic $\omega-3$
PUFA found in plants is $\alpha$-linolenic acid (18:3) (LA), which can be synthesised in the body to the longer-chain, more polyunsaturated $\omega$-3 PUFAs known as eicosapentanoic acid (20:5) (EPA) and docosohexanoic acid (22:6) (DHA) by the action of desaturase enzymes. These same desaturase enzymes convert the $\omega-6$ PUFAs, another essential PUFA, to arachidonic acid (AA) and thus compete with the $\omega-3$ series. A high ratio of dietary linoleic acid ( $\omega$-6) to linolenic acid ( $\omega$ 3 ) is likely to produce the greatest depletion of the longerchain $\omega$-3 PUFAs such as EPA and DHA.

Fish is the best dietary source of $\omega$-3 PUFAs (DHA, EPA) and world-wide prevalence of depression has been significantly correlated with low fish consumption ${ }^{1}$. In this comparative study, New Zealand was found to have one of the highest rates of major depression and lowest consumption of fish per capita in the world. In addition, there are fewer oily fish species living in the waters of the Southern Hemisphere.

The aim of the present study was to assess whether selfreported mental health status, measured by the Mental 
Health scale of the SF-36 questionnaire in a sample of 4644 New Zealand adults, was associated with fish consumption (measured with a food-frequency questionnaire).

\section{Method}

\section{Survey design}

The data consist of the combined 1996/97 New Zealand Health Survey and 1997 Nutrition Survey, which were conducted using the same sampling frame. The health survey was administered first and the nutrition survey later to the $80 \%$ of health survey respondents who agreed to take part in the nutrition survey. The SF-36 was administered as part of the health survey, and the questions about fish consumption formed part of the nutrition survey.

The sampling frame was a clustered, stratified design based on contingent geographic areas called Primary Sampling Units (PSUs), each containing 50 to 100 dwellings. The PSUs were divided into 122 strata, according to various characteristics derived from the 1991 Census data. The sample was drawn from all 122 strata, to ensure a sample with characteristics representative of the total population. The sample consisted of 11921 households with a final adult sample size of 7862 for the health survey (response rate of $73.8 \%$ ) and 4644 for the nutrition survey (effective response rate of 50\%). Survey participants completed the SF-36 questionnaire themselves, after having taken part in the health survey faceto-face interview which collected sociodemographic information, information related to healthcare utilisation, the prevalence of certain medical conditions (e.g. asthma, diabetes, hypertension) and risk-related behaviours (e.g. smoking, level of physical activity, alcohol use). Further details on the survey methodologies can be found elsewhere ${ }^{12,13}$.

The SF-36 is a generic, health-related quality of life questionnaire originally developed in the United States ${ }^{14}$ and since used widely in a number of other countries ${ }^{15-17}$. The SF-36 consists of 36 items, grouped into eight scales, each 0-100 scale measuring a different aspect of health; higher scale scores represent better self-reported health. The scales are: Physical Functioning; Role Physical (the impact of physical health on performance of everyday role); Bodily Pain; General Health; Vitality; Social Functioning; Role Emotional (the impact of emotional health on role performance); and Mental Health. Additionally, two summary measures can be calculated based on principal component factor score coefficients. The summary scores represent aggregate measures of those scales most correlated with the underlying dimensions of physical health (the Physical Component Summary score (PCS)) and mental health (the Mental Component Summary score (MCS) $)^{18}$.

The measures used here were the two scale scores that are the best single indices of self-reported physical health and mental health status (Physical Functioning and Mental Health), together with the two summary scores, PCS and MCS. The latter are standardised to a mean of 50 and standard deviation of 10, whereas the scale scores are not standardised and have standard deviations of 22 and 15 (in the New Zealand population), respectively. This means that group differences on the scale scores appear larger than group differences on the summary scores.

\section{Statistical analysis}

The data were analysed using the SUDAAN® (Research Triangle Institute) statistical package. The use of SUDAAN enables appropriate computation of standard errors and variance estimates from cluster-correlated data. All analyses applied a survey weight (uniquely assigned to each respondent), which adjusted for varying probabilities of selection among members of the sample population, and post-stratified the age and sex distribution of the sample so that it matched the age and sex distribution of the New Zealand population.

The independent variable was fish consumption. Survey respondents were categorised into those who consumed no fish of any kind and those who consumed some kind of fish, at any frequency (ranging from less than once per month to two or more times per day). 'Fish' could include canned tuna, salmon, sardines, eel, fish battered, fried, steamed, baked, grilled or raw, shellfish, or other seafood.

The adjusted least-square mean scores of the Physical Functioning and Mental Health scales, and of the PCS and MCS summary measures, for the two fish consumption groups were obtained using the Proc Regress function of SUDAAN. Hierarchical models were constructed, first with adjustment for age (four groups: 15-24 years; 25-44 years; 45-64 years; 65+ years) and annual household income (four groups: <\$20000; \$20001-30000; \$30 001-50 000; \$50000+). Smoking status (smokers, exsmokers and non-smokers) and alcohol use (nondrinkers, moderate drinkers (scoring $1-7$ on AUDIT*) and potential problem drinkers (scoring $8+$ on AUDIT)) were then additionally adjusted for, as these have been associated with lower levels of $\omega$-3 PUFAs in tissues ${ }^{19,20}$. Eating patterns (meat eaters, vegetarians, vegan) were also adjusted for as this variable, not surprisingly, differed across consumption groups (more vegetarians and vegans among non-fish eaters) and may be a marker for a generally 'healthier' lifestyle. Other demographic variables such as gender, ethnicity and education, together with potentially confounding nutrient factors - such as the intake of iron, selenium, niacin, vitamin $\mathrm{B}_{12}$, cholesterol, total fat and total energy, were included in the preliminary analyses. As none of these variables was found to have a

\footnotetext{
*Alcohol Use Disorders Identification Test: a World Health Organization (WHO) screening instrument designed to identify those at risk of developing psychological or physical problems from alcohol consumption, referred to here as 'potential problem drinkers'.
} 
Table 1 Means scores on SF-36 scales and summary measures

\begin{tabular}{|c|c|c|c|c|}
\hline Fish consumption & $\begin{array}{l}\text { Mental Health } \\
\text { scale }\end{array}$ & $\begin{array}{l}\text { Physical Functioning } \\
\text { scale }\end{array}$ & $\begin{array}{l}\text { Mental Component } \\
\text { Score }\end{array}$ & $\begin{array}{l}\text { Physical Component } \\
\text { Score }\end{array}$ \\
\hline \multicolumn{5}{|c|}{ Adjusted for age and household income } \\
\hline Yes $(n=4557)$ & 78.8 & 86.5 & 50.5 & 50.0 \\
\hline No $(n=87)$ & $69.9^{\star \star \star}$ & 89.7 & $42.9^{\star \star \star}$ & 50.5 \\
\hline \multicolumn{5}{|c|}{ Adjusted for age, household income, smoking and alcohol consumption, and eating pattern } \\
\hline Yes $(n=4557)$ & 78.8 & 86.6 & 50.5 & 50.0 \\
\hline No $(n=87)$ & $70.6^{* *}$ & $91.0^{*}$ & $43.0^{* * *}$ & 52.2 \\
\hline
\end{tabular}

${ }^{*}, P<0.05 ;{ }^{* *}, P<0.01$; ${ }^{* * *}, P<0.001$; significant difference across fish consumption groups.

significant relationship with fish consumption, they were not included in the final model.*

\section{Results}

\section{Fish consumption groups and SF-36 scales}

After adjustment for age and income, but before adjustment for smoking, alcohol use and eating pattern, a significant association was found between fish consumption and the Mental Health scale (Wald $F(1$, $8)=10.97, P=0.0009)$. The adjusted means for the two fish consumption groups are presented in Table 1. These show that the adjusted mean Mental Health scale score is significantly lower in the group who consume no fish (low scores representing poorer mental health). By contrast, the adjusted mean for the Physical Functioning scale shows a trend (Wald $F(1,8)=3.77, P=0.052$ ) in the opposite direction, i.e. a higher score among those who consume no fish. As Table 1 also shows, the same pattern occurred using the principal component summary scores, with a significantly lower MCS score (Wald $F(1,8)=13.34$, $P=0.0003$ ) among the non-fish consumers and no difference across fish consumption groups on PCS (Wald $F(1,8)=0.07$, not significant (NS)). Because these summary scores are standardised to have a mean of 50 , they are useful for interpreting the group difference: namely, that it is the non-fish consumers who are scoring significantly lower on the mental health measures, rather than the fish consumers who are scoring significantly higher.

After additional adjustment for smoking, alcohol use and eating pattern, the difference in scores between fish consumption groups on the Mental Health scale (Wald $F(1,14)=7.81, P=0.005)$ and MCS summary scores (Wald $F(1,14)=10.62, P=0.001$ ) was reduced slightly, but remained significant. The two groups now differed

\footnotetext{
*The distributions of the SF-36 scales were far from normal, and the distributions of the summary scores were also not quite normal, although they were closer to normality. After transformation of the summary scores using a negative log transformation, normality was achieved, and the significance of the association between fish consumption and MCS was increased, as was the $R^{2}$ value for the model. In the interests of clarity, however, the untransformed data and results are reported below.
}

significantly on the Physical Functioning scale, with nonfish consumers scoring higher $(P=0.045)$

One significant interaction was found: between age group and fish consumption, whereby the relationship between fish consumption (or lack of it) and mental health scores occurred only in the two younger age groups (1524 years and $25-44$ years). However, these two groups also comprised most of the non-fish-consuming group (62 out of 87). Additionally, the older two age groups did not show any tendency towards an opposite effect (i.e. higher mental health scores in non-fish consumers) so it is considered that the main effect reported above, associating lack of fish consumption with lowered mental health scores, remains valid.

Further analyses were undertaken to investigate whether different categorisation of the fish consumption groups altered the pattern of effects. For example, fish consumption was categorised into those who consumed some kind of fish at least once a week and those who consumed it less often (including not at all). However, comparison of the mental and physical health scores (using either the scales or summary scores) of these two groups found no significant difference. Similarly, comparison of those who consumed a lot of fish (more than one kind of fish at least twice a week) with those who consumed less also found no significant results. Additionally, there was no significant correlation (Spearman) between frequency of fish consumption and mental health or physical functioning scores.

\section{Discussion}

This is the first population survey study to show a significant relationship between fish intake and selfreported mental health status in adults. The results of this work are generally supportive of other findings that fish consumption is inversely correlated with depression ${ }^{1}$.

It is likely that this association between self-reported mental health status and fish consumption is mediated by $\omega-3$ PUFAs found in relatively high concentrations in fish. This would support work by others which shows that concentrations of $\omega-3$ PUFAs are significantly lower in the red blood cell membranes of depressed patients compared with controls ${ }^{2,3}$.

Biochemical mechanisms provide a theoretical basis for 
the possible effect of $\omega$ - 3 PUFAs on mood and depression because changes in the phospholipid composition of cell membranes, particularly related to DHA content, alter membrane microstructure and the function of membraneassociated proteins, such as enzymes, transport proteins and receptors ${ }^{21-24}$.

However, before conclusions are drawn, it is important to consider whether the statistical differences in selfreported mood, as measured by the SF-36 scores between fish consumption groups, represent differences of clinical or public health significance. Ware and co-workers ${ }^{25}$ provide a guideline that a difference of 5 points between groups on an SF-36 scale, corresponding to an effect size of around 0.2 (the expression of group differences in standard deviation units), is small but 'clinically and socially significant'. The difference reported here for the Mental Health scale is around 9 points, equivalent to an effect size of 0.6, which is usually classified as a moderate difference $^{26}$. For the MCS score, the difference of around 7 points is an even greater effect size of 0.7. These differences can also be put in context by comparison with the magnitude of differences obtained from the larger health survey sample between groups categorised by other factors, where differences as large as 8 or 9 points on the Mental Health scale or 7 points on the MCS score were seldom, if ever, observed. For example, the (age- and sexstandardised) difference between lowest and highest household income groups was 5.2 for the Mental Health scale and 2.7 for MCS; the corresponding figures for highest and lowest alcohol consumption groups were 4.2 and 2.0; and for labour force status groups (employed vs. unemployed) the difference was 5.1 and 2.9. In this light, the differences observed here are substantial.

In interpreting these results, a second consideration is the relationship between the lower scores on the SF-36 mental health scales, found here among non-fish eaters, and clinical depression. The SF-36 Mental Health scale is not a measure of depression, but it does tap mood state. So it can be safely stated that those who have lower scores on the Mental Health scale do have lower mood, although it is not possible to determine what proportion of those individuals may have a mood disorder (clinical depression). However, if depressed mood is considered from a continuum rather than a categorical perspective, then the relationship between SF-36 scores and fish consumption may be very important for a population. It may be the case, for example, that if fish consumption (or more specifically $\omega-3$ PUFAs) is influential in low mood, and low mood is one of the precursors of a clinical state of depression, then level of $\omega$-3 PUFAs may be a component of biological vulnerability that may help explain why some individuals shift from low mood into a state of clinical depression or have difficulty getting out of a state of depression. These are issues for further research.

Clearly, the number of non-fish eaters in this study is very small relative to the fish-eating group, raising the possibility that the significantly lower self-reported mental health scores in non-fish eaters are an aberration, stemming from the small sample or from poor health generally amongst that group. This highlights the difficulty of determining the direction of effect in cross-sectional survey analysis. For example, it could be the case that depressed mood leads to a more restricted diet, and consequently poorer health. The key result that argues against such an interpretation, however, is that there was either no difference in self-reported Physical Functioning scores across fish consumption groups or a difference in the opposite direction (higher Physical Functioning scores in the non-fish eating group). This supports the main interpretation that the lower Mental Health scale scores of the non-fish eating group are a function of fish consumption itself, although this cannot be proved statistically in this study.

Furthermore, when the data were adjusted for other possible confounding variables such as sociodemographic factors, health risk behaviours (such as smoking and alcohol use), eating patterns and those nutrients found in relatively high concentrations in fish and hypothesised to be relevant to mental health (iron, selenium, niacin, vitamin $\mathrm{B}_{12}$, cholesterol, total fat intake and total energy intake), the significant relationship between mental health status and fish consumption remained.

As described, the nutrition survey 'piggy-backed' on the health survey and as a result the response rate for the health survey (74\%) was greater than that for the nutrition survey (50\%), which raises the question of possible sample bias. However, a comparison of the demographic information of participants considered to be related to diet showed that the two survey samples were very similar, suggesting that the dropout between the two surveys was non-differential ${ }^{27}$. It remains a possibility that calculated nutrient variables might be biased towards a healthy population, which may overestimate nutrient intakes. However, this is unlikely to limit the validity of the present study because individual nutrient intakes were related to an individual's self-reported mental health status and hence any association will remain valid.

A possible dose-response relationship between fish or $\omega-3$ PUFA consumption and self-reported mental health status or depression is not yet known. From this study it would appear that consumption of fish less than once a month is sufficient for improved self-reported mental health status. This is supported by evidence that DHA has a half-life of 21 days in the brain ${ }^{28}$ and may indicate that very small amounts are required to keep cell membrane levels 'topped up' enough to prevent a change in mood, which may then lead to depression. Similarly significant differences are often observed only between fish eaters and non-fish eaters when assessing the risk of coronary disease $^{29}$. This is to be expected, as the association is likely to be strongest with a long-standing dietary practice, given the limitations of dietary assessment ${ }^{30}$, and in view of the 
fact that the assessments of dietary intake and mental health status in the present study were performed on different occasions.

We conclude that this is the first cross-sectional national survey to demonstrate a significant relationship between fish intake and self-reported mental health status, which is consistent with the hypothesis that $\omega$-3 PUFAs may act as mood stabilisers in human health. Experimental evidence is now required to determine whether the link is causal, whether the mediating factors are indeed $\omega-3$ PUFAs, and whether changing dietary behaviour to increase intakes of fish and or $\omega-3$ PUFAs can improve self-reported mental health status and reduce the incidence of depression in the 21st century.

\section{Acknowledgements}

The surveys on which this study was based were funded by the New Zealand Ministry of Health.

\section{References}

1 Hibbeln J. Fish consumption and major depression. Lancet 1998; 351: 1213.

2 Peet M, Murphy B, Shay J, Horrobin D. Depletion of omega-3 fatty acid levels in red blood cell membranes of depressive patients. Biol. Psychiat. 1998; 43: 315-9.

3 Edwards R, Peet M, Shay J, Horrobin D. Omega-3 polyunsaturated fatty acid levels in the diet and in red blood cell membranes of depressed patients. J. Affect. Disord. 1998; 48: 149-55.

4 Stoll AL, Severus WE, Freeman MP, et al. Omega 3 fatty acids in bipolar disorder: a preliminary double-blind, placebo controlled trial. Arch. Gen. Psychiat. 1999; 56: 407-12.

5 Cross-National Collaborative Group. The changing rate of major depression. J. Am. Med. Assoc. 1992; 268: 3098-150.

6 Klerman GL, Weissman MM. Increasing rates of depression. J. Am. Med. Assoc. 1989; 261: 2229-35.

7 Taylor TG, Gibney MJ, Morgan JB. Homeostatic function and polyunsaturated fatty acids. Lancet 1979; 2: 1378.

8 Eaton MD, Konner M. Paleolithic nutrition: a consideration of its nature and current implications. N. Engl. J. Med. 1985; 312: 283-9.

9 Leaf A, Weber PC. A new era for science in nutrition. Am. J. Clin. Nutr. 1987; 45: 1048-53.

10 Budowski P. $\omega 3$-Fatty acids in health and disease. World Rev. Nutr. Diet. 1988; 57: 214-74.

11 Booth-Kewley S, Friedman HS. Psychological predictors of heart disease: a quantitative review. Psychol. Bull. 1987; 101: 343-62.

12 Ministry of Health. Taking the Pulse: The 1996/97 New Zealand Health Survey. Wellington: Ministry of Health, 1999.
13 Quigley R, Watts C. Food Comes First: Methodologies for the National Nutrition Survey of New Zealand. Wellington: Ministry of Health, 1997.

14 Ware JE, Sherbourne CD. The MOS 36-item short-form health survey (SF-36) I. Conceptual framework and item selection. Med. Care 1992; 30: 473-83.

15 Sullivan M, Karlson J, Ware J. The Swedish SF-36 health survey. I. Evaluation of data quality, scaling assumptions, reliability and construct validity across general populations in Sweden. Soc. Sci. Med. 1995; 42: 1349-58.

16 Ware JE, Kosinski M, Gandek B, et al. The factor structure of the SF-36 health survey in 10 countries: results from the IQOLA project. J. Clin. Epidemiol. 1998; 51: 1159-65.

17 Scott KM, Tobias M, Sarfati D, Haslett SJ. SF-36 Health Survey reliability validity and norms for New Zealand. Aust. NZ J. Public Health 1999; 23: 401-6.

18 Ware JE, Kosinski M, Keller SD. SF-36 Physical and Mental Health Summary Scales: A User's Manual. Boston, MA: The Health Institute, 1994.

19 Simon JA, Fong J, Bernert JT, Browner WS. Relation of smoking and alcohol consumption to serum fatty acids. Am. J. Epidemiol. 1996; 144: 325-34.

20 Pawlosky RJ, Salem N Jr. Alcohol consumption in rhesus monkeys depletes tissues of polyunsaturated fatty acids and alters essential fatty acid metabolism. Alcohol Clin. Exp. Res. 1999; 23: 311-7.

21 Salem N, Niebylski C. The nervous system has an absolute requirement for proper function. Mol. Membr. Biol. 1995; 12: 131-4.

22 Litman BJ, Mitchell DC. A role for phospholipid polyunsaturation in modulating membrane protein function. Lipids 1996; 31: S193-7.

23 Slater SJ, Kelly MB, Yeager MD, et al. Polyunsaturation in cell membranes and lipid bilayers and its effects on membrane proteins. Lipids 1996; 31: S189-92.

24 Witt MR, Westh-Hansen SE, Rasmussen PB, et al. Unsaturated free fatty acids increase benzodiqazepine receptor against binding depending on the subunit composition of GABA(A) receptor complex. J. Neurochem. 1996; 67: 2141-5.

25 Ware JE, Snow KK, Kosinski M, Gandek B. SF-36 Health Survey. Manual and Interpretation Guide. Boston, MA: The Health Institute, 1993.

26 Kazis LE, Anderson JJ, Meenan RF. Effect sizes for interpreting changes in health status. Med. Care 1989; 27: S178-89.

27 Russell DG, Parnell WR, Wilson NC. Nutrition trends in New Zealand. New Zealand Public Health Rep. 1999; 6: 65-8.

28 Conner WE, Neuringer M, Lin DS. Dietary effects on brain fatty acid composition: the reversibility of $\omega-3$ fatty acid deficiency and turnover of docosahexaenoic acid in the brain, erythrocytes, and plasma of rhesus monkeys. J. Lipid Res. 1990; 31(2): 237-47.

29 Ascherio A, Rimm EB, Stampfer MJ, et al. Dietary intake of marine $\omega-3$ fatty acids, fish intake, and the risk of coronary disease among men. N. Engl. J. Med. 1995; 332: 977-82.

30 Willett WC. Epidemiologic studies of diet and cancer. Prog. Clin. Biol. Res. 1990; 346: 159-68. 